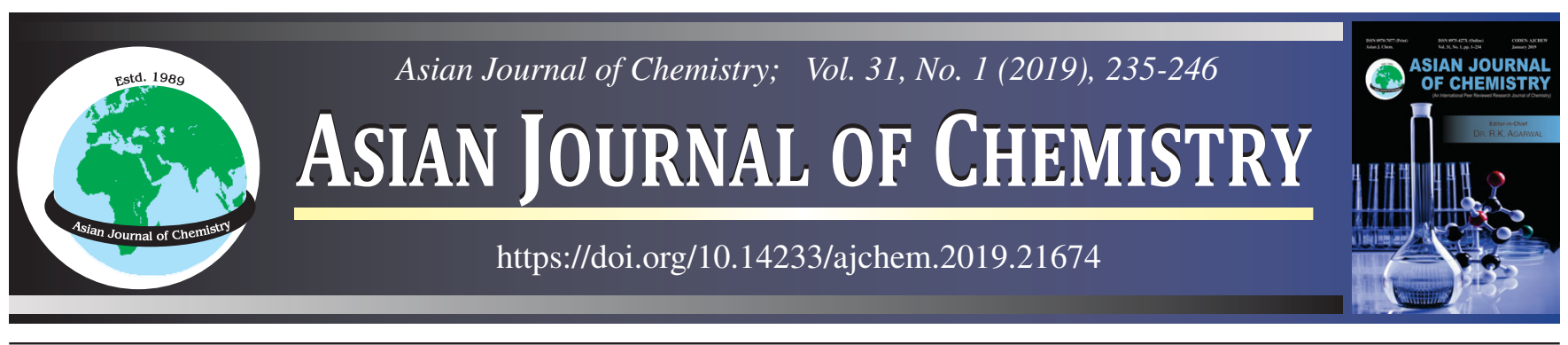

\title{
Poly(styrene) Beads Grafted with Dendrimer Stabilized Gold Nanoparticles for Catalytic Reduction of 4-Nitrophenol
}

\section{Paramasivam Shanmugam ${ }^{1,2}$, Wei Wei ${ }^{1,3}$, Jimin XiE $^{1, *}$ and Eagambaram Murugan ${ }^{4, *}$}

${ }^{1}$ School of Chemistry and Chemical Engineering, Jiangsu University, Zhenjiang 212013, P.R. China

${ }^{2}$ Department of Chemistry, St. Joseph University, Dimapur-797115, India

${ }^{3}$ Center of Analysis and Test, Jiangsu University, Zhenjiang 212013, P.R. China

${ }^{4}$ Department of Physical Chemistry, School of Chemical Sciences, University of Madras, Chennai-600025, India

*Corresponding author: E-mail: shanmugachem@gmail.com

Received: 25 August 2018; Accepted: 22 October 2018; Published online: 30 November 2018;

Four types of new bead-shaped heterogeneous nanoparticle catalysts viz., polymer-supported poly(styrene) beads grafted with poly(propylene imine) dendrimer(G2) (PPI-G2) stabilized gold nanoparticles (AuNPs) viz., PS-PVBC-PPI(G2)-AuNPs-L \& PS-P4VP-PPI(G2)-AuNPsL and PS-PVBC-PPI(G2)-AuNPs-H \& PS-P4VP-PPI(G2)-AuNPs-H were prepared by varying the metal load $5.7 \times 10^{-3} \mathrm{mM}($ low metal load $-\mathrm{L}$ ) and $11.410^{-3} \mathrm{mM}$ (high metal load $-\mathrm{H}$ ) respectively. Initially, two different insoluble matrices including poly(styrene)-copoly(vinylbenzyl chloride) (PS-PVBC) grafted with PPI(G2) and poly(styrene)-co-poly(4-vinylpyridine) (PS-P4VP) grafted with PPI(G2) dendrimer were prepared individually by suspension polymerization method and then the epoxy group was generated on both matrix via atom transfer radical polymerization (ATRP) and quaternization methods. The resulting matrixes grafted with PPI(G2) was stabilized individually with the addition of $\mathrm{HAuCl}_{4}$ and thus obtained four types of new heterogeneous nanoparticle catalysts. These catalysts were characterized with UV-visible, FTIR, SEM, HRTEM \& EDS techniques and their catalytic potential were examined by reduction of 4nitrophenol as a model reaction under pseudo first order reaction condition. The obtained rate constant $\left(\mathrm{k}_{\mathrm{obs}}\right)$ values revealed that the catalysts derived from ATRP method viz., PS-PVBC-PPI(G2)-AuNPs-H and PS-PVBC-PPI(G2)-AuNPs-L has shown 6 and 4 fold active than the catalysts derived from quaternized method viz., PS-P4VP-PPI(G2)-AuNPs-H and PS-P4VP-PPI(G2)-AuNPs-L respectively. The reusability of the superior catalyst was verified for the same reaction and proved the activity was sustained up to fourth cycle.

Keywords: Polymer beads, Atom transfer radical polymerization, Heterogeneous nanoparticles, Dendrimer, 4-Nitrophenol.

\section{INTRODUCTION}

The application of nanotechnology in the field of catalysis has created a remarkable impact in advancement of chemistry, because catalysis is the central field of chemical science. In general, the surface of the catalyst plays a major role in accelerating the chemical reaction irrespective of homogeneous or heterogeneous catalysts $[1,2]$. In general, the synthesis of heterogeneous nanoparticle catalyst involves immobilization of metal nanoparticles on various solid matrices. The more often used solid supports for immobilization of metal nanoparticles are inorganic materials like silica [3,4], alumina [5,6], zeolites [7], metal oxides [8], carbon [9] and organic materials like polymer-supported polymer beads [10-14], polymer resin [2]. Particularly, research on stabilization/immobilization of metal nanoparticles onto the functionalized insoluble polymer-beads as matrix was started earlier $[15,16]$ and now it has received a greater attention everywhere and growing very fast [13]. In the preparation of heterogeneous nanoparticle catalyst, the functionalization/grafting of specific or desired functional groups onto the surface of insoluble polymer-bead matrix is generally a deciding factor for efficient stabilization/ immobilization of metal nanoparticles to produce an efficient catalyst. In spite of the fact that many functional modifications were performed already on the surface of cross-linked polymersupported poly(styrene) beads matrix $[17,18]$, the conventional type of insoluble bead-shaped polymer-supported matrices are not popular for efficient stabilization/immobilization of metal nanoparticles and hence have not received much attention among the catalytic industries. The main reason for this is due

This is an open access journal, and articles are distributed under the terms of the Creative Commons Attribution-NonCommercial 4.0 International (CC BY-NC 4.0) License, which allows others to copy and redistribute the material in any medium or format, remix, transform, and build upon the material, as long as appropriate credit is given and the new creations are licensed under the identical terms. 
to the lesser availability of functional groups onto the surface of matrix and hence poorest immobilization of metal nanoparticles.

Alternatively, researchers have prepared insoluble polymer-supported bead-shaped matrices with more functional groups viz., hydrazine [19] and poly(hydroxyethyl acrylate) (HEA) [20] on the surface by surface initiated atom transfer radical polymerization technique (SI-ATRP) and used the same for greater immobilization of invertase enzyme and heavy metal ions uptakes. The critical advantage of this ATRP technique is provided the controlled chain growth, well defined block, graft copolymer and particularly, formation of homopolymer is completely eliminated, thus enabling the generation of more functional group onto the surface of the matrix for free stabilization/immobilization of metal nanoparticles catalyst [21]. Hence, it is proved that SI-ATRP technique is considered to be very efficient one for grafting of more functional groups onto solid surfaces such as nanomaterial, fibers, films, membranes etc. [22].

For the past two decades we have been continuously reporting various bead-shaped polymer-supported phase transfer catalysts $[23,24]$ and thus application for various organic reactions with better yield. In the earlier studies, we have developed bead-shaped poly(styrene) surface-enriched activesite polymer-supported phase transfer catalyst and their corresponding nano metal immobilized heterogeneous nanoparticle catalysts. However, the matrices for these catalysts were prepared through free radical polymerization technique. Further, using our continuous experience gained in this field, we firmly believed that, in free radical polymerization technique, the number of functional group generated onto the surface of beadshaped poly(styrene) matrix is always lesser and particularly while on constructing the polymer supported matrix, certain amount of functional monomer was buried inside the matrix and some functional monomer has undergone homo polymerization and thus the possibility to bring more surface functional group onto the matrices is very difficult.

Recently, we have reported polymer-supported bead-shaped heterogeneous nanoparticle catalysts with homogeneous distribution of well-defined gold nanoparticles using PPI(G2) dendrimer as a stabilizing agent [14]. In this preparation, initially, the poly(styrene) supported poly(vinyl benzyl) chloride (PS-PVBC) was prepared by suspension polymerization technique using AIBN as a free radical initiator. The stabilizing agent viz., PPI $(\mathrm{G} 2)$ was then functionalized onto the matrix by surface grafting technique followed by stabilization/immobilization of metal nanoparticles. The obtained catalyst was studied for the reduction of nitrophenol. Here also, it is observed that the extent of PPI $(\mathrm{G} 2)$ grafted onto the PS-P4VP matrices was not sufficient and thus only average number of stabilization/ immobilization of metal nanoparticles onto the surface was noticed in the corresponding catalyst. Further, there is no report available for the preparation of bead-shaped insoluble polymer-supported poly(styrene) matrix that contain more functional groups, particularly achieved through SI-ATRP for efficient stabilization/ immobilization of metal nanoparticles catalysts. Furthermore, it is well known that 4-aminophenol (4-AP) is a potential intermediate in the manufacturing of analgesic and antipyretic drugs such as paracetamol, phenacetin etc., [25,26].
Similarly, among several water pollutants, nitro aromatic compounds are considered to be the most toxic and refractory pollutants. Specifically, nitrophenols based solvents were used for building blocks of many dyes, explosives and pesticides $[27,28]$ and thus their effluents contributed heavy pollutions to water and soil. More particularly, dye industry contaminating local water bodies and also as potent uncouples in oxidative and photosynthetic phosphorylation [29]. In order to degrade the nitrate pollutants more effectively, various types of research have been performed already in the field of catalysis and different types of heterogonous polymer-supported nanoparticle catalysts were developed. Wherein, we understand from the literature studies that, although many reports are available for preparation of polymer-supported matrices for development of heterogeneous nanoparticles catalyst, only few reports are available on the development of polymer-supported beads grafted with dendrimer as a stabilizing agent. Under these circumstances, we observed that it is necessary to prepare a stable, more efficient, bead-shaped heterogeneous nanoparticle catalyst with intensively distributed metal nanoparticles onto the surface of the matrix.

Therefore, in the present study 4 types of polymer-supported heterogeneous nanoparticles catalysts were prepared using two different types of cross-linked co-polymer beads viz., poly(styrene)-co-poly(vinyl benzyl chloride) (PS-PVBC) and poly(styrene)-co-poly(4-vinyl pyridine) (PS-P4VP) as individual matrices and on each matrix two different load of catalytic moiety viz., $\mathrm{HAuCl}_{4}$ was immobilized. All these catalysts were prepared by adopting the four steps methods. In the first step, PS-PVBC and PS-P4VP beads were prepared by following the literature procedure through suspension polymerization method. In second step, glycidyl methacrylate was functionalized onto the PS-PVBC polymer beads via surface initiatedatom transfer radical polymerization (SI-ATRP) and then epichlorohydrin was functionalized onto PS-P4VP via simple quaternization reaction method thus yielding the corresponding epoxy functionalized polymer beads labeled as PS-PVBCGMA and PS-P4VP-ECH, respectively. In the third step, PPI(G2) dendrimer was grafted on both the matrices viz., PS-PVBC-GMA and PS-P4VP-ECH and thus produced PS-PVBC-GMA-PPI(G2) and PS-P4VP-PPI(G2). In the fourth step, immobilization/stabilization of AuNPs onto the PS-PVBC-PPI(G2) and PS-P4VP-PPI(G2) matrices was performed individually by taking two different $\left[\mathrm{HAuCl}_{4}\right]$ and thus produced two heterogeneous nanoparticle catalysts from each matrices which includes, PS-PVBC-PPI(G2)-AuNPs with lower metal load \& PS-P4VP-PPI(G2)-AuNPs with lower metal load and PS-PVBC-PPI(G2)-AuNPs with higher metal load \& PS-P4VP-PPI(G2)-AuNPs with higher metal load. Then the catalytic potential of these catalysts was inspected through reduction of 4-nitrophenol performed under pseudofirst order condition.

\section{EXPERIMENTAL}

Styrene (Aldrich), vinyl benzyl chloride (VBC), divinylbenzene (DVB) (Lancaster), gelatin (SD fine), boric acid (SRL), poly(vinyl alcohol) (SRL), sodium hydroxide (SRL), sodium nitrite (SRL), sodium sulfate (SD fine), AIBN (SRL), 
auric acid (SRL), ethanol (SRL), epichlorohydrin (SRL), 4nitrophenol (SRL), sodium borohydride (SD fine), poly(propylene imine) dendrimer generation-2 (PPI-G2) (symo-chem, Netherland), glycidyl methacylate (GMA), phenanthracene and $\mathrm{CuBr}$ were received from Sigma Chemical and all analar grade (99\% purity) and used as received. Double distilled water was used for solutions preparation.

Purification method for the monomers: The monomers were purified by treating twice with $\mathrm{NaOH}(10 \%$ w/v) so as to remove the inhibitor viz., 4-tert-butylcatechol (TBC) and subsequently washing three times with distilled water and then the supernatant was transferred to column packed with dried $\mathrm{K}_{2} \mathrm{CO}_{3}$ over the aluminum oxide as adsorbent. Finally, the purified monomer eluted from column was dehydrated using anhydrous sodium sulfate and then used for polymerization reaction.

Characterization: FT-IR analysis was carried out on BRUKER, TENSOR 27 model instrument. UV-visible absorption spectrum was measured on Perkin Elmer UV-visible spectrophotometer. The background correction was made by absorption spectrum of water for catalytic studies. The surface morphology of the bead-shaped polymer supported matrix and their corresponding nanoparticle catalysts were analyzed by scanning electron microscope (SEM). The measurements were performed through HITACHI S-3000H scanning electron microscope accelerated at $20 \mathrm{keV}$ with different working distance. The sample preparation for analysis was carried out by the following method. That is, a strip of double-sided conducting carbon tape was applied to the metal stub on which the sample to be analyzed. The respective sample was scanned with HRTEM using JEOL 3010 high-resolution transmission electron microscope (HRTEM) operated at $300 \mathrm{keV}$.

Preparation of two types of bead-shaped polymer supported matrices viz., poly(styrene)-co-poly(vinylbenzyl chloride) (PS-PVBC) and poly(styrene)-co-poly(4- vinylpyridine) (PS-P4VP): Insoluble cross linked polymer-supported poly(vinyl benzyl chloride) (PS-PVBC) beads were prepared by adopting the earlier method [30] and (Scheme-I) by fixing the functional monomer (vinyl benzyl chloride) load as $25 \%$, cross linking monomer divinyl benzene (DVB) load as $3 \%$ and remaining load (72\%) as a supporting monomer viz., styrene (St). The organic phase was maintained at $82.5 \mathrm{~g}$ and the aqueous phase was kept at $225 \mathrm{~g}$. Exactly, 1.35g of gelatin, $2.55 \mathrm{~g}$ of boric acid and $2.25 \mathrm{~g}$ of poly (vinyl alcohol) were dissolved in 35,60 and $130 \mathrm{~mL}$ of double distilled hot water $\left(50^{\circ} \mathrm{C}\right)$, respectively. Then, these solutions were mixed together at room temperature and the viscosity of the medium was maintained by poly(vinyl alcohol). The $\mathrm{pH}$ of the resultant solution was adjusted to 10 by adding $25 \%$ of aqueous sodium hydroxide $(\mathrm{w} / \mathrm{v})$ and then $0.1 \mathrm{~g}$ of sodium nitrite was added to maintain the $\mathrm{pH}$. The aqueous phase became clear only after the $\mathrm{pH}$ was adjusted to 10. It was then transferred into $500 \mathrm{~mL}$, three-necked round bottom flask equipped with an overhead mechanical stirrer and a reflux condenser. Nitrogen gas was passed continuously and the temperature was fixed at $50{ }^{\circ} \mathrm{C}$. After $0.5 \mathrm{~h}$, with the aforementioned conditions maintained, the organic phase containing $3.3 \mathrm{~g} \mathrm{(2 \% )}$ of DVB, $58.57 \mathrm{~g}$ of styrene and $0.4125 \mathrm{~g}$ of AIBN, was thoroughly mixed and added to the reaction flask. The blade level of the stirrer was adjusted so that the tips were in the organic phase and most of the blade was in aqueous phase. Subsequently, the thermostat temperature was increased to 70 ${ }^{\circ} \mathrm{C}$ and the stirring speed was maintained at $400 \mathrm{rpm}$ with a tachometer. The co-polymerization of styrene and divinyl bezene was allowed to proceed for up to $3 \mathrm{~h}$. After $3 \mathrm{~h}$, vinylbenzylchloride (functional monomer) was added. This reaction was continued for $36 \mathrm{~h}$ after which the reaction mixture washed with hot water and ethanol and dried at $60{ }^{\circ} \mathrm{C}$ in vacuum.

Similarly, the second category of insoluble cross-linked poly(styrene) beads functionalized with 4-vinyl pyridine was obtained by fixing the $25 \%$ of 4 -vinyl pyridine load (functional monomer) and $3 \%$ as a crosslinking load and the rest is styrene and thus produced the cross-linked poly(styrene)-co-poly(4vinyl pyridine) (PS-P4VP) bead matrices. However, the only variation is instead of using vinylbenzyl chloride as a functional monomer, here 4-vinyl pyridine was used as a functional monomer and as usual the divinyl benzene and styrene were used as a cross-linking and supporting monomers, respectively. The dried beads were sieved into different mesh sizes, the respective mesh size of (+100 to 120) were used as matrices irrespective of the types and were made into corresponding heterogeneous nanoparticles catalyst.

Functionalization of glycidyl methacrylate (GMA) onto PS-PVBC beads via surface initiated-atom transfer radical polymerization (SI-ATRP) technique: Glycidyl methacrylate (GMA) was functionalized onto the PS-PVBC beads by following the surface initiated-atom transfer radical polymerization method (SI-ATRP) $[19,20]$. To state briefly, PS-PVBC beads $(5 \mathrm{~g})$ were transferred into a $100 \mathrm{~mL}$ round bottom flask and swelled in ethanol for $24 \mathrm{~h}$. To that $20 \mathrm{~mL}$ of GMA (0.15 mol), $0.432 \mathrm{~g}$ of $\mathrm{CuBr}(3.0 \mathrm{mmol}), 0.936 \mathrm{~g}$ of $1,10-$ phenantracene $(6.0 \mathrm{mmol})$ and $10 \mathrm{~mL}$ of dioxane were added and the reaction mixture was stirred at $65^{\circ} \mathrm{C}$ for $18 \mathrm{~h}$ under $\mathrm{N}_{2}$ atmosphere. The resulting GMA functionalized PS-PVBC beads were filtered and transferred into a $100 \mathrm{~mL}$ round bottom flask containing $50 \mathrm{~mL}$ of EDTA ( $10 \% \mathrm{w} / \mathrm{v})$ and it was stirred continuously for $24 \mathrm{~h}$ to remove the excess copper ions. The resulting beads were washed sequentially with hot water, ethanol and dried in vacuum oven at $60{ }^{\circ} \mathrm{C}$ for $24 \mathrm{~h}$ and thus yielded GMA grafted poly (vinyl benzyl chloride) beads which were labeled as PS-PVBC-GMA (Scheme-I). The PS-PVBCGMA beads were characterized with FTIR and SEM and the observed spectra and images are shown in Figs. 1 and 3, respectively.

Surface grafting of PPI(G2) dendrimer onto PS-PVBCGMA beads via epoxy ring opening reaction: Initially, PSPVBC-GMA beads $(0.5 \mathrm{~g})$ were taken in a $100 \mathrm{~mL}$ round bottom flask and swelled with 2-methyl pyrrolidone $(20 \mathrm{~mL})$ for $24 \mathrm{~h}$. PPI(G2) dendrimer $(50 \mathrm{mg}$ ) was added and the reaction mixture was stirred at room temperature for $24 \mathrm{~h}$ and again stirred for $5 \mathrm{~h}$ at $90{ }^{\circ} \mathrm{C}$. After completion of the reaction, the mixture was allowed for cooling, washed with hot water and methanol to remove the unreacted dendrimer and the resulting beads were dried under vacuum at $60{ }^{\circ} \mathrm{C}$ for $24 \mathrm{~h}$ to yield PPI(G2) grafted PS-PVBC-GMA beads labeled as PS-PVBCPPI(G2) (Scheme-I). These beads in turn were characterized with FTIR and SEM analyses and the observed spectra and images are shown in Figs. 1 and 3, respectively. 

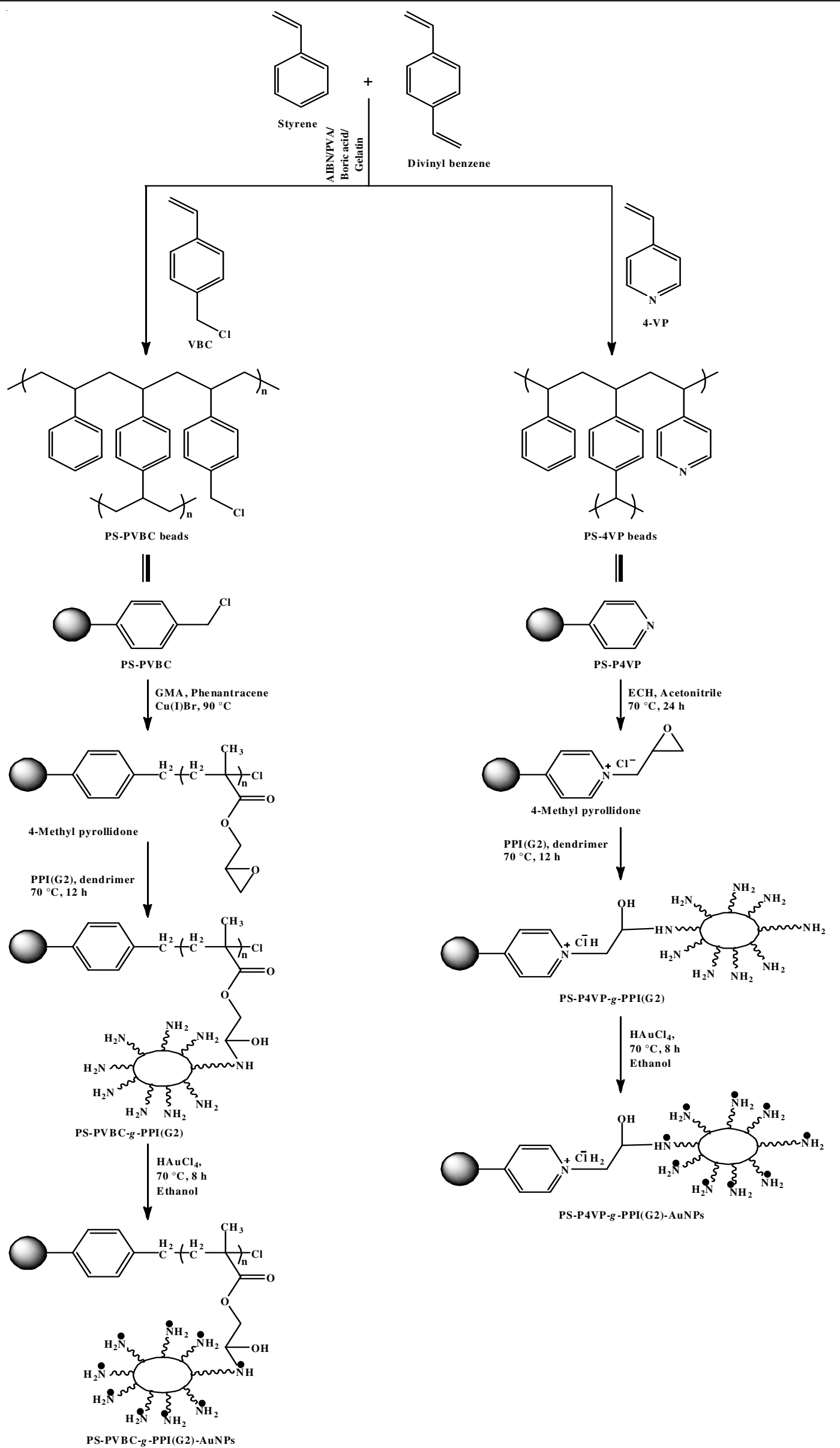

Scheme-I: Schematic representation of synthesis of four different types of polymer supported nanoparticle catalysts 


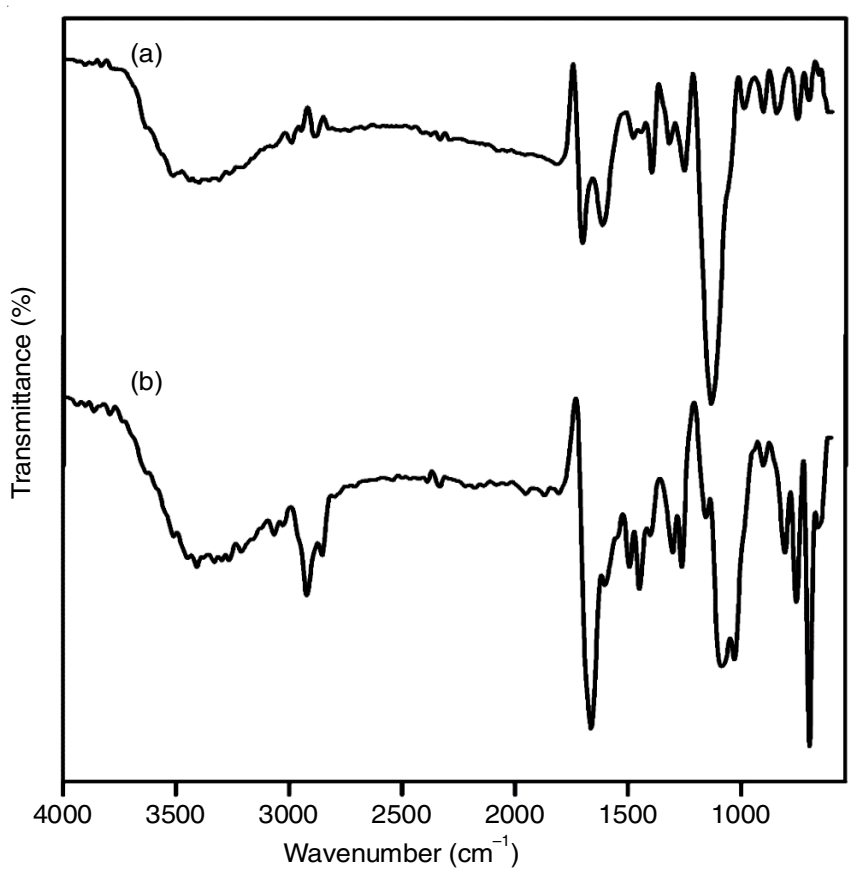

Fig. 1. FTIR spectrum of (a) PS-PVBC-GMA, (b) PS-PVBC-PPI(G2)

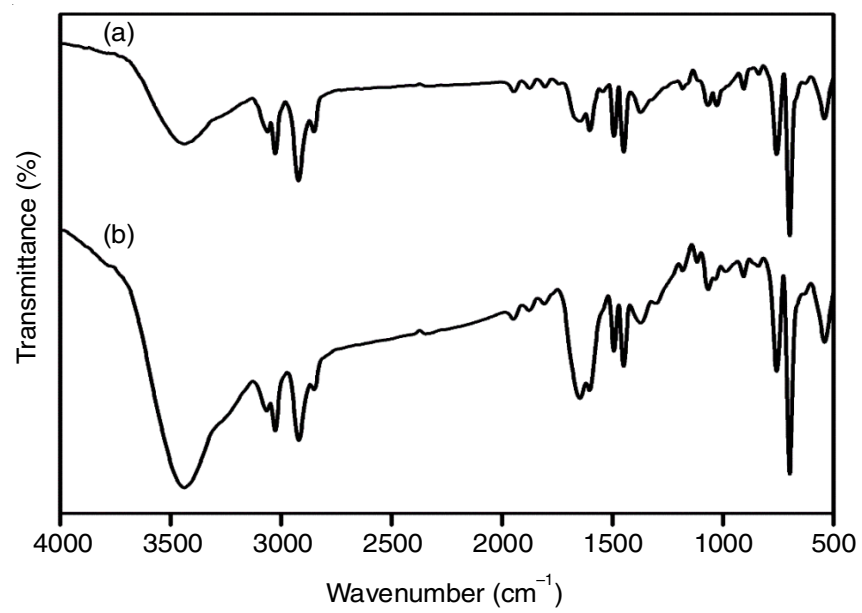

Fig. 2. FTIR spectrum of (a) PS-P4VP-GMA, (b) PS-P4VP- PPI(G2)

Functionalization of epichlorohydrin (ECH) onto the PS-P4VP beads via simple quaternization reaction: Epichlorohydrin $(\mathrm{ECH})$ was functionalized onto the PS-P4VP beads by following the simple quaternization reaction $[19,20]$. PSP4VP beads $(5 \mathrm{~g})$ were taken in $50 \mathrm{~mL}$ round bottom flask and swelled in $10 \mathrm{~mL}$ THF. To that, $1 \mathrm{~mL}$ of epichlorhydrin was added under continuous stirring and the reaction mixture was stirred for $24 \mathrm{~h}$ at $60^{\circ} \mathrm{C}$ to yield epichlorohydrin grafted poly(4vinyl pyridine) beads labeled as PS-P4VP-ECH (Scheme-I). The resulting PS-P4VP-ECH beads were characterized by FTIR and SEM analyses and the observed spectrum and images are shown in Figs. 2 and 4, respectively.

Surface grafting of PPI(G2) dendrimer PS-4VP-ECH beads via epoxy ring opening reaction: Initially, $0.5 \mathrm{~g}$ of PS-P4VP-ECH beads were taken in a $100 \mathrm{~mL}$ round bottom flask containing PPI $(\mathrm{G} 2)$ dendrimer $(50 \mathrm{mg})$ and 2-methyl pyrrolidone $(20 \mathrm{~mL})$ and the reaction mixture was stirred at room temperature for $24 \mathrm{~h}$ and then again stirred for $5 \mathrm{~h}$ at 90 ${ }^{\circ} \mathrm{C}$. After completion of the reaction, the mixture was allowed
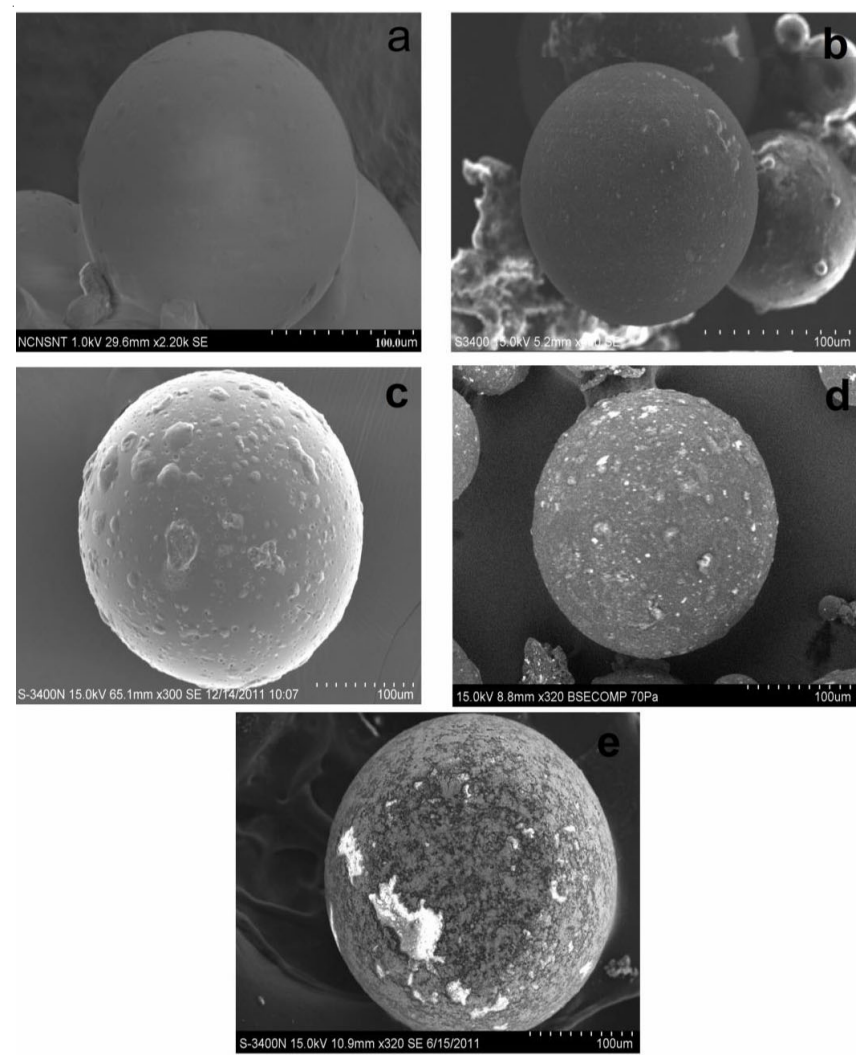

Fig. 3. SEM images of (a) PS-PVBC, (b) PS-PVBC-GMA, (c) PS-PVBCPPI(G2), (d) PS- PVBC-PPI(G2)-AuNPs-L catalyst, (e) PS-PVBCPPI(G2)-AuNPs-H catalyst
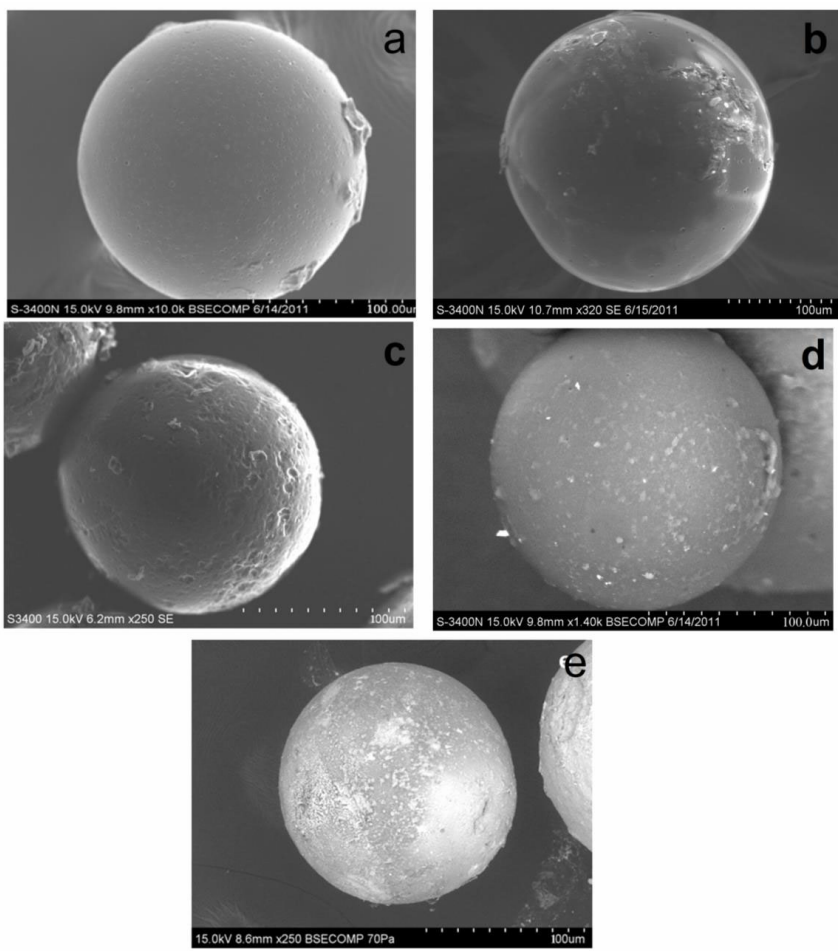

Fig. 4. SEM images of (a) PS-P4VP, (b) PS-P4VP-ECH, (c) PS-P4VPPPI(G2) (d) PS- P4VP-PPI(G2)-AuNPs-L catalyst (e) PS-P4VPPPI(G2)-AuNPs-H catalyst

for cooling, then it was subsequently washed with hot water and methanol to remove the unreacted dendrimer and the resulting beads were dried under vacuum at $60^{\circ} \mathrm{C}$ for $24 \mathrm{~h}$ to yield 
PPI(G2) grafted PS-P4VP-ECH beads labeled as PS-P4VPPPI(G2) (Scheme-I). The beads were characterized with FTIR and SEM and the observed spectrum and images were shown Figs. 2 and 4, respectively.

Preparation of 4 types of bead shaped new heterogeneous nanoparticle catalysts via stabilization of different load of AuNPs onto the PS-PVBC-PPI-(G2) and PSP4VPPPI (G2) matrices: First two types of new bead-shaped heterogeneous nanoparticle catalysts were prepared individually via stabilization of $5.7 \times 10^{-3} \mathrm{mM}$ of $\mathrm{HAuCl}_{4}$ load onto the PSPVBC- PPI(G2) and PS-P4VP-PPI(G2) matrices (Scheme-I) and thus produced corresponding low metal loaded catalysts viz., PS-PVBC-PPI(G2)-AuNPs-L \& PS-P4VP-PPI(G2)AuNPs-L. That is, $100 \mathrm{mg}$ of PS-PVBC-PPI(G2) and PS-P4VPPPI(G2) bead matrices were taken individually in two different $25 \mathrm{~mL}$ conical flask and suspended in $10 \mathrm{~mL}$ of solvent mixture ( $7.5 \mathrm{~mL} \mathrm{H}_{2} \mathrm{O} / 2.5 \mathrm{THF}$ ) for $6 \mathrm{~h}$. To that, $5.7 \times 10^{-3} \mathrm{mM}$ of $\mathrm{HAuCl}_{4}$ $(2.25 \mathrm{mg})$ was added in both containers maintained at room temperature and the respective solution was stirred for $1 \mathrm{~h}$. As a result, the polymer beads present in each container were turned from pale yellow to intense yellow and then gradually changed into red, indicating the stabilization of $\mathrm{Au}^{3+}$ onto the PPI(G2) dendrimer grafted onto the surface of the respective polymer matrices. After $1 \mathrm{~h}, \mathrm{Au}^{3+}$ stabilized complex viz., PSPVBC-PPI(G2)-Au ${ }^{3+}$ and PS-P4VP-PPI(G2)-Au ${ }^{3+}$ were filtered, washed with water and methanol and then dried. Then these complex beads such as PS-PVBC-PPI(G2)-Au ${ }^{3+}$ and PS-P4VP$\mathrm{PPI}(\mathrm{G} 2)-\mathrm{Au}^{3+}$ were taken individually in $50 \mathrm{~mL}$ round bottom flask and subjected to reduction by the addition of $1 \mathrm{mmol}$ of cold aqueous $\mathrm{NaBH}_{4}$ solution ( $10 \mathrm{~mL}$ ) and the respective solution was stirred vigorously for about $2 \mathrm{~h}$ at room temperature. After the reduction of $\mathrm{Au}^{3+}$, the red colour of the respective beads had completely turned into dark brown. The product was washed with ethanol and dried under vacuum at $60^{\circ} \mathrm{C}$ for $24 \mathrm{~h}$, the respective bead-shaped heterogeneous nanoparticle catalysts labeled as PS-PVBC-PPI(G2)-AuNPs-L and PSP4VP-PPI(G2)-AuNPs-L. Similarly, another two types of same insoluble heterogeneous nanoparticle catalysts were prepared by stabilizing the higher load of $\mathrm{HAuCl}_{4}$ i.e. $11.4 \times 10^{-3} \mathrm{mM}$ $(4.50 \mathrm{mg})$ individually onto same PS-PVBC-PPI $(\mathrm{G} 2)$ and PSP4VP-PPI(G2) matrices. Otherwise the procedure followed for preparation of earlier two catalysts were also exactly the same and thus produced another two types of new bead-shaped heterogeneous nanoparticle catalysts which were labeled as PS-PVBC-PPI(G2)-AuNPs-H and PS-P4VP-PPI(G2)-AuNPs$\mathrm{H}$. All the 4 types of new bead-shaped heterogeneous nanoparticles catalysts viz., PS-PVBC-PPI(G2)-AuNPs-L \& PSP4VP-PPI(G2)-AuNPs-L and PS-PVBC-PPI(G2)-AuNPs-H \& PS-P4VP-PPI(G2)-AuNPs-H were characterized with UVvisible, HRTEM, SEM and EDAX analyses.

Comparative catalytic potential of 4 types of heterogeneous catalyst with 4-nitrophenol: The comparative catalytic potential of all the 4 types of catalysts was studied individually by performing the reduction of 4-nitrophenol as a model reaction under pseudo first order reaction condition (Scheme-II). That is, irrespective of the catalyst, the reaction was performed in quartz cuvette (having path length of $1 \mathrm{~cm}$ ) kept at ambient temperature. Exactly $0.25 \mathrm{~mL}$ of $(1 \mathrm{mmol})$ aqueous sodium borohydride solution, $0.25 \mathrm{~mL}(0.01 \mathrm{mmol})$ of aqueous of 4nitrophenol were taken in a cuvette to which $2.5 \mathrm{~mL}$ double distilled water was added. $6 \mathrm{mg}$ of catalyst was added into the medium. The progress of reaction was monitored quantitatively by UV-visible spectrophotometry by measuring the decrease in absorbance at the characteristic peak viz., $-\mathrm{NO}_{2}$ at $400 \mathrm{~nm}$ (for 4-nitrophenolate ion). The time required for complete reduction of specific concentration of the substrate was determined. To test the stability of the superior catalyst viz., PS-PVBC-PPI(G2)AuNPs-H, the catalyst used in the first cycle was filtered off from the reaction medium and reused again for upto 4 cycles by maintaining similar reaction conditions and the respective time required for the complete reduction of substrate was noted and compared with the time observed in the first cycle.

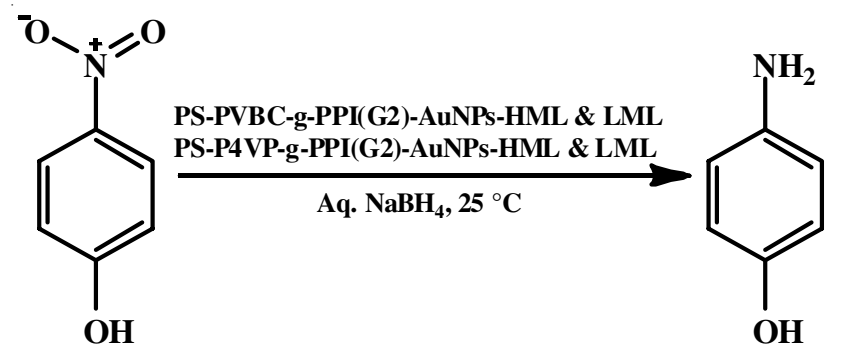

4-Nitrophenol

4-Aminophenol

Scheme-II: Reduction of 4-nitrophenol

RESULTS AND DISCUSSION

The matrices such as PS-PVBC and PS-P4VP were prepared initially by mixing the respective functional monomer viz., either vinylbenzylchloride or 4-vinylpyridine simultaneously with styrene, divinylbenzene and then ter-polymerization reaction in aqueous/organic phase was performed by suspension polymerization technique. It is shown from these studies that the concomitant addition of functional monomer along with supporting/cross linking (styrene + divinyl benzene) monomers irrespective of matrix causes most of the functional group to be buried inside the matrix and thereby it may not available for reaction. Hence, the proportion of functional group available on the surface of bead matrix is low. Hence, in the subsequent studies [23], styrene and divinyl benzene are pre-polymerized and then the functional monomer vinylbenzyl chloride was added by delayed addition method and this method has relatively brought better amount of functional group on the matrix than the simultaneous addition of functional monomer method. We observed that this method also not effective an inferior one to bring more functional group onto the surface. However, in the present study PS-PVBC and PS-P4VP matrices are prepared individually by following the delayed addition strategy of functional monomers vinylbenzyl chloride and 4-vinyl pyridine onto the respective pre-polymerized monomers of styrene/divinyl benzene by $3 \mathrm{~h}$ through suspension polymerization technique. Then their individual dried beadshaped polymer-supported matrices were used as a precursor to graft intensive surface functional group. It is more important to mention here that in order to improve the strength/stability of the respective matrix the cross-linked monomer load viz., divinyl benzene was fixed as $3 \%$. 
FTIR spectroscopy: The FTIR spectra of PS-PVBC and PS-P4VP gives the characteristic peak at $698 \mathrm{~cm}^{-1}$ for $\mathrm{C}_{-} \mathrm{Cl}_{(\mathrm{str})}$ and at $1372 \mathrm{~cm}^{-1}$ for aromatic $\mathrm{C}-\mathrm{N}_{\text {(str) }}$ thus indicating the functionalization of vinyl benzyl chloride and 4-vinyl pyridine in the respective matrix. Then, the glycidyl methacylate was grafted on PS-PVBC matrix via SI-ATRP method and thus obtained PS-PVBC-GMA bead matrix. The FTIR of this PS-PVBCGMA matrix shows the intense characteristic peaks at 1704 $\mathrm{cm}^{-1}$ due to $\mathrm{C}=\mathrm{O}_{\text {(str) }}, 1254 \mathrm{~cm}^{-1}$ and $905.64 \mathrm{~cm}^{-1}$ for symmetric and asymmetric vibrations respectively of epoxy group (Fig. 1a). Appearance of these peaks reveals the functionalization/ grafting of glycidyl methacylate on PS-PVBC. The grafting of stabilizing agent [viz., PPI(G2) dendrimer] in PS-PVBCPPI(G2) matrix was established through the appearance of new peaks at $3066 \mathrm{~cm}^{-1}$ and $1653 \mathrm{~cm}^{-1}$ which corresponds to $-\mathrm{NH}_{2 \text { (str) }}$ and $-\mathrm{N}-\mathrm{H}$ deformation, respectively in FTIR (Fig. 1b) and these characteristic peaks are obviously due to surface amines and internal tertiary amines of PPI(G2) (Fig. 1b).

In the case of PS-P4VP matrix, the linker group viz., epichlorohydrin was functionalized onto the matrix by following the simple quarterization reaction. The functionalization of epichlorohydrin in PS-P4VP-ECH is justified through its FTIR (Fig. 2a), in which appearance of new characteristic peaks at $1180 \mathrm{~cm}^{-1}$ and $905 \mathrm{~cm}^{-1}$ are due to symmetric and asymmetric vibrations respectively of epoxy ring and also the peak observed at $1492 \mathrm{~cm}^{-1}$ is due to aromatic ring of 4-vinylpyrine and thus indicating the functionalization of epichlorohydrin and 4-vinyl pyridine in PS-P4VP-ECH matrix. On the contrary, the FTIR of PS-4VP-PPI(G2) has shown (Fig. 2b), new peaks at $3280 \mathrm{~cm}^{-1}$ and $1634 \mathrm{~cm}^{-1}$ due to -NH (str) and N-H (bend) for secondary amine group in PPI(G2) and these confirm the grafting of PPI(G2) onto PS-P4VP-ECH.

SEM and EDAX analyses: The surface morphologies of PS-PVBC, PS-PVBC-GMA, PS-PVBC-PPI(G2) and PSPVBC-PPI(G2)-AuNPs-L \& PS-P4VP-PPI(G2)-AuNPs-L and PS-PVBC-PPI(G2)-AuNPs-H \& PS-P4VP-PPI(G2)-AuNPs$\mathrm{H}$ catalysts were studied with SEM and the obtained images are shown in Figs. 3a-e and 4a-e, respectively. From the images, it is observed that the SEM images of plain matrices viz., PSPVBC (Fig. 3a) and PS-P4VP (Fig. 4a) show spherical in shape with homogenous smooth surface, whereas the images of PSPVBC-GMA (Fig. 3b) and PS-P4VP-ECH (Fig. 4b) reveal that the surface of the former contained more severe heterogeneity than the latter one, indicating that the glycidyl methacylate is grafted onto the surface of PS-PVBC matrix due to SI-ATRP technique. In contrast, the mild heterogeneity noticed in the surface of the latter one is due to average grafting of epichlorohydrin in PS-P4VP-ECH matrix due to simple quaternization reaction. Further increment of surface heterogeneity/roughness noticed in Fig. 3d indicates the intensive grafting of PPI $(\mathrm{G} 2)$ onto the PS-PVBC-GMA-PPI(G2) and average grafting of PPI(G2) PS-P4VP-ECH-PPI (G2) matrices. The formation and stabilization of AuNPs on to the PS-PVBC-PPI(G2) and PSP4VP-PPI(G2) matrices were confirmed by respective SEM images shown in Fig. 3d and Fig. 4d. Here also more intensively distributed tiny dots are observed in the surface of the former one. This is because, in PS-PVBC-PPI (G2)-AuNPs, the polymer support viz., PS-PVBC-GMA was prepared via ATRP method thus leading to functionalization of ' $n$ ' number of glycidyl methacrylate unit onto the surface of the PS-PVBC beads and thereby increased grafting of more number of PPI $(G 2)$ dendrimer onto the PS-PVBC-GMA matrix [19]. Further, on stabilization of different [AuNPs] on the matrix viz., PS-PVBCPPI (G2) and PS-P4VP-PPI (G2), the previous one is enable to stabilized more number of AuNPs due to free availability of more $-\mathrm{NH}_{2}$ group than the latter one and hence the surface of PS-PVBC-PPI(G2)-AuNPs has shown intensively distributed dots than PS-P4VP-PPI (G2)-AuNPs irrespective of the variation of $\left[\mathrm{HAuCl}_{4}\right]$. Similarly, among the SEM images of high and low metal loaded catalysts, irrespective of the type of matrix, the surface of the high metal loaded catalysts show more dots reflecting increased stabilization of metal nanoparticles (Figs. $3 \mathrm{e}$ and $4 \mathrm{e}$ ).

The grafting of PPI $(\mathrm{G} 2)$ dendrimer onto the surface of PSPVBC-GMA and PS-P4VP-ECH matrices and their corresponding ability for stabilization of metal nanoparticles were studied extensively with EDAX. The characteristic elements such as $\mathrm{C}, \mathrm{N}, \mathrm{O}, \mathrm{Cl}$ and $\mathrm{Au}$ and their corresponding quantity (\%) available before and after stabilization of AuNPs onto the respective matrix are given in Table-1. The comparative results reveal that the percentage of $\mathrm{O}, \mathrm{N}$ and $\mathrm{Cl}$ is relatively higher in plain PS-PVBC-PPI(G2) than with PS-P4VP-PPI(G2). The enhanced percentage of oxygen noticed in the first matrix is attributed to the availability of more epoxy group due to grafting of glycidyl methacylate, leading to increased grafting of more PPI(G2). This result in a higher percentage of nitrogen from surface amine group and finally more $-\mathrm{Cl}$ is available from pendant benzyl chloride group from vinyl benzyl chloride. Therefore, the availability of more functional group such as glycidyl methacylate, $\mathrm{PP}(\mathrm{G} 2)$ and pendant benzyl chloride achieved onto surface of PS-PVBC-PPI(G2) matrix is solely responsible for intensive grafting of the said group due to SI-ATRP technique. In contrast, in PS-P4VP-PPI(G2) matrix, the surface grafting of epichlorohydrin and PPI(G2) was performed through simple quaternization reaction and thereby observed decreased $\%$ of oxygen due to less number of epoxy group from epichlorohydrin and thereby lesser grafting of PPI(G2) which is noticed from lesser percentage of nitrogen from surface $-\mathrm{NH}_{2}$ group.

TABLE-1

PERCENTAGE OF ELEMENTS DETERMINED FROM EDAX ANALYSIS USING POLYMER SUPPORTED AuNPS CATALYST

\begin{tabular}{lccccc}
\hline \multirow{2}{*}{ Name of the compound } & \multicolumn{4}{c}{ Value of elements $(\%)$ presents in PS-PVBC-PPI(G2)-AuNPs \& PS-PVBC-PPI(G2)-AuNPs } \\
\cline { 2 - 6 } & $\mathrm{C}$ & $\mathrm{N}$ & $\mathrm{O}$ & $\mathrm{Cl}$ & $\mathrm{Au}$ \\
\hline PS-PVBC-PPI(G2) & 38.12 & 56.64 & 3.12 & 2.12 & - \\
PS-P4VP-PPI(G2) & 55.02 & 41.05 & 2.41 & 2.52 & - \\
PS-PVBC-PPI(G2)-AuNPs -L & 68.41 & 23.17 & 4.32 & 3.90 & 1.20 \\
PS-PVBC-PPI(G2)-AuNPs -H & 71.46 & 17.76 & 2.06 & 2.12 & 1.71 \\
PS-P4VP-PPI(G2)-AuNPs -L & 65.02 & 29.91 & 3.41 & 2.41 \\
PS-P4VP-PPI(G2)-AuNPs -H & 67.14 & 26.29 & & 0.44 \\
\hline
\end{tabular}


Obviously, the percentage of chloride is also less irrespective of the matrix, the rest of the percentage are carbon. Therefore, as explained in SEM results, the SI-ATRP technique is proved to be effective for binding more number of GMA/PPI $(\mathrm{G} 2)$ group onto the surface of PS-PVBC matrix than with simple quaternization reaction occurred on PS-P4VP-PPI(G2) matrix. Similarly, irrespective of low and higher metal loaded catalysts, the percentage of AuNPs noticed in PS-PVBC-PPI(G2) matrix are observed to be largely higher than their counter part matrix based catalyst i.e., PS-P4VP-PP(G2). Of course, it is obviously known that the greater extents of stabilization of AuNPs noticed in the previous matrix are directly related to availability of $-\mathrm{NH}_{2}$ group onto the surface, thus confirming earlier inferences. In the case of higher percentage of AuNPs observed in high metal loaded catalyst again reflect the increased stabilization of AuNPs than their corresponding low metal loaded catalysts.

UV-visible and HRTEM analysis: The stabilization of AuNPs onto the respective 4 types of matrices were also inspected through UV-visible and HRTEM analyses. To know the stabilization of AuNPs onto the matrices, all the four types of heterogeneous nanoparticle catalysts were separately taken in $100 \mathrm{~mL}$ round bottom flask and were swelled with $50 \mathrm{~mL}$ of ethanol. $5 \mathrm{mg}$ of cetyl trimethyl ammonium bromide (CTAB) was added to each flask for extraction of AuNPs from the respective matrix $[13,14,31]$. Then the respective catalyst solution was sonicated for $1 \mathrm{~h}$ and then the corresponding filtrate was characterized by UV-visible and HRTEM analyses. The recorded UV-visible spectra for PS-PVBC-PPI(G2)-AuNPs-L \& PSP4VP-PPI(G2)-AuNPs-L and PS-PVBC-PPI(G2)-AuNPs-H \& PS-P4VP-PPI(G2)-AuNPs-H catalysts are shown in Figs. 5a-b and 6a-b, respectively. The appearance of SPR peaks at $536 \mathrm{~nm}$ indicates the formation and stabilization of AuNPs onto the surface of the respective matrices. Similar results were reported the synthesis of heterogeneous catalyst of polymersupported poly(propylene imine) dendrimer gold nanoparticles [14]. The PS-PVBC-PPI(G2)-AuNPs-L \& PS-PVBCPPI(G2)-AuNPs-H catalysts have shown increased SPR peak

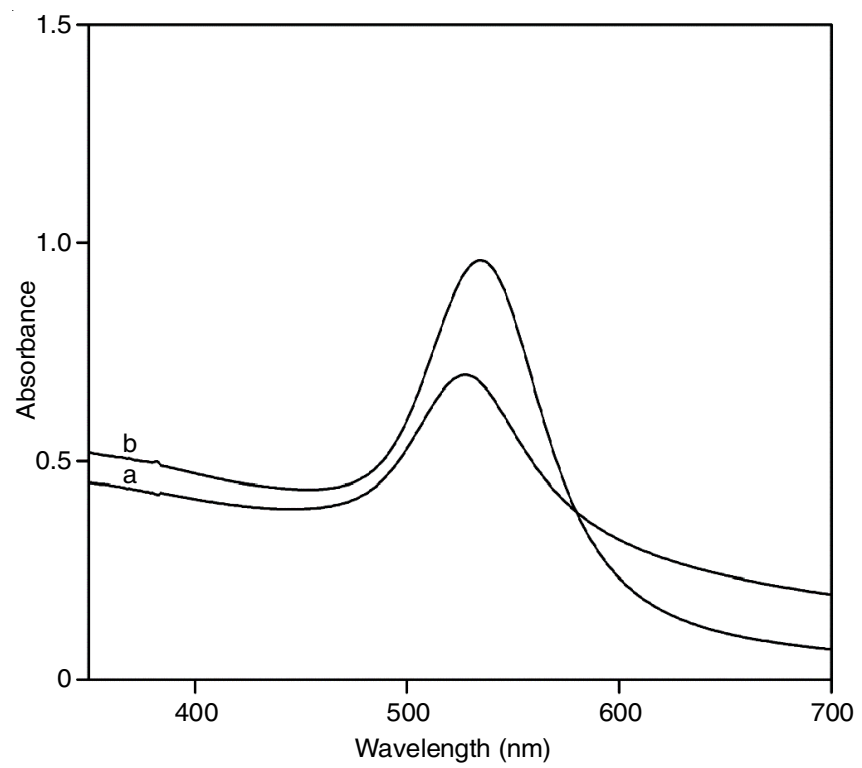

Fig. 5. UV-visible spectra of (a) PS-PVBC-PPI(G2)-AuNPs-L catalyst, (b) PS-PVBC- PPI(G2)-AuNPs-H catalyst

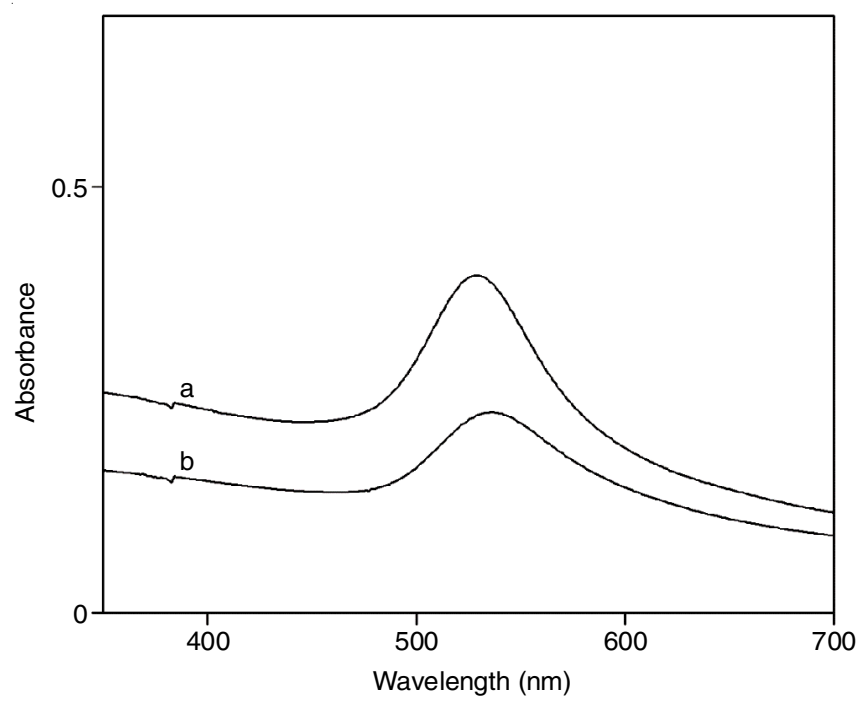

Fig. 6. UV-visible spectra of (a) PS-P4VP-PPI(G2)-AuNPs-L catalyst, (b) PS-P4VP-PPI(G2)-AuNPs-H catalyst

intensity as compared with PS-P4VP-PPI(G2)-AuNPs-L \& PSP4VP-PPI(G2)-AuNPs-H catalyst. This observation further confirms the stabilization of AuNPs onto the PS-PVBC-PPI(G2) and thus possible because SI-ATRP technique.

Further, these findings were again verified with results obtained from HRTEM analysis. The obtained HRTEM images for representative catalysts viz., PS-PVBC-PPI(G2)-AuNPs$\mathrm{H} \&$ PS-P4VP-PPI(G2)-AuNPs-H are shown in Fig. 7a and $b$. On comparing Fig. $7 \mathrm{a}$ and $\mathrm{b}$ it was found that the solution extracted from and PS-PVBC-PPI(G2)-AuNPs-H catalyst contains more block dots than the solution extracted from PSP4VP-PPI(G2)-AuNPs catalyst. Therefore, it is necessary to highlight that during the synthesis of catalysts, even though equal amount of dendrimer was added to both the matrices, due to the SI-ATRP technique we have generated more amount of epoxy groups onto the PS-PVBC beads and thus enabled the grafting of more amount of $\mathrm{PPI}(\mathrm{G} 2)$ dendrimer onto the surface of PS-PVBC-GMA matrix compared with quaternized PS-P4VP-ECH matrix. Hence, obviously the influence noticed in HRTEM strongly shows that more number of AuNPs was stabilized onto the surface of PS-PVBC-PPI(G2) than with the PS-P4VP-PPI $(\mathrm{G} 2)$ and the size of the AuNPs was found to be in the range of 15 to $20 \mathrm{~nm}$.

Comparative catalytic efficiency of all the four types of catalysts: The comparative catalytic efficiency among the four catalysts was assessed based on the on the $\mathrm{k}_{\text {obs }}$ determined for reduction of 4-nitrophenol under identical reaction condition. As mentioned earlier, the occurrence of the reaction was monitored through UV. From the UV-visible spectrum, the intensity of absorption peak observed at $400 \mathrm{~nm}$ for $\mathrm{NO}_{2}$ group found to decrease gradually. Subsequently, there is a gradual development of the new absorption peak at $300 \mathrm{~nm}$ $\left(\mathrm{NH}_{2}\right)$ was conforming the conversion of 4-nitrophenol to 4-amino phenol (Fig. 8). The observed rate constants (Table2) reveal that the irrespective of higher and lower metal loaded catalysts derived from ATRP has showed substantial increment nearly four times active than their corresponding higher and lower metal loaded catalysts derived from quaternization method viz., PS-P4VP-PPI(G2)-AuNPs-H \& PS-P4VP-PPI(G2)- 

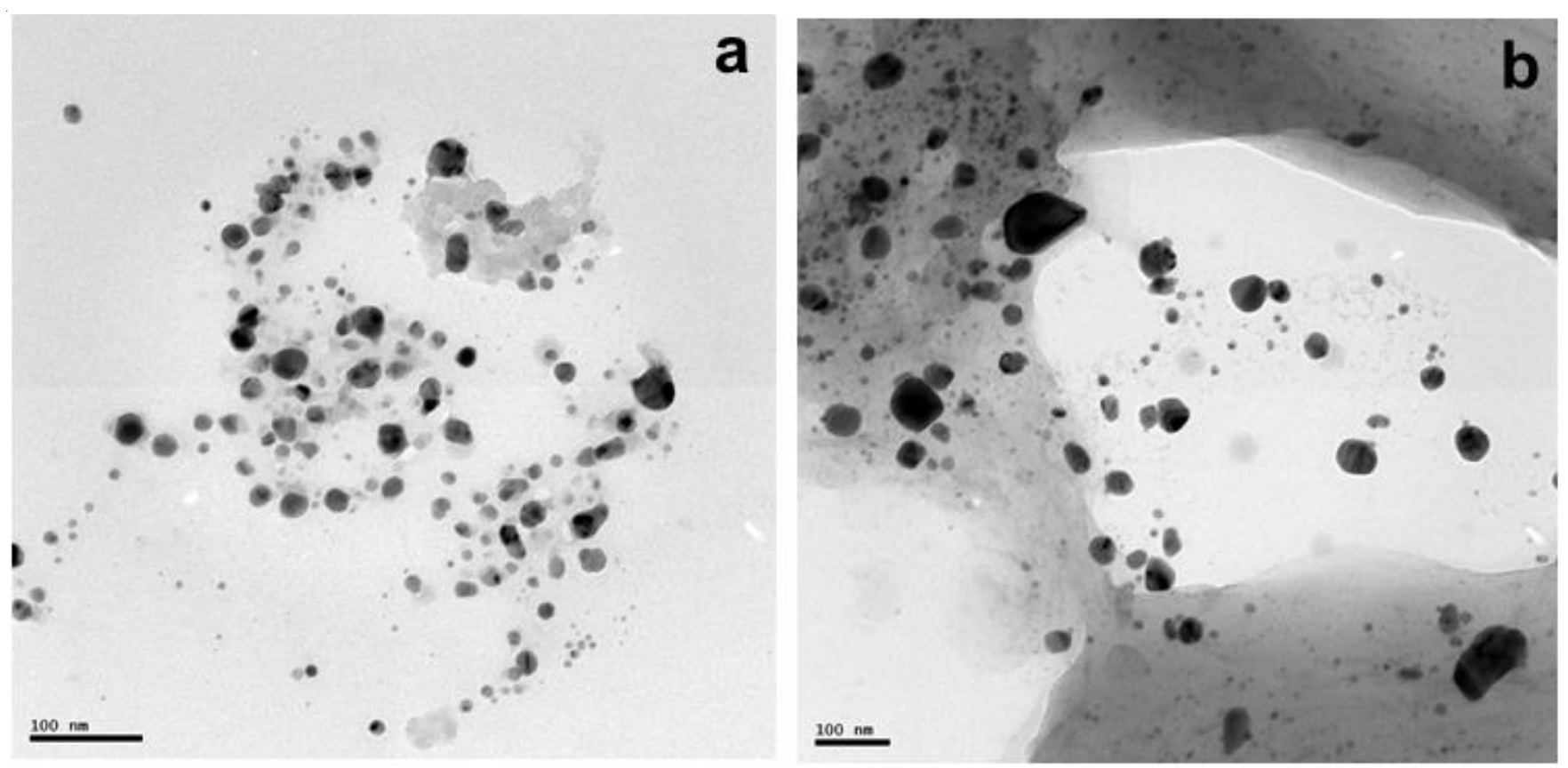

Fig. 7. HRTEM images of (a) and PS-PVBC-PPI(G2)-AuNPs-H catalyst, (b) PS-P4VP-PPI(G2)-AuNPs-H catalyst

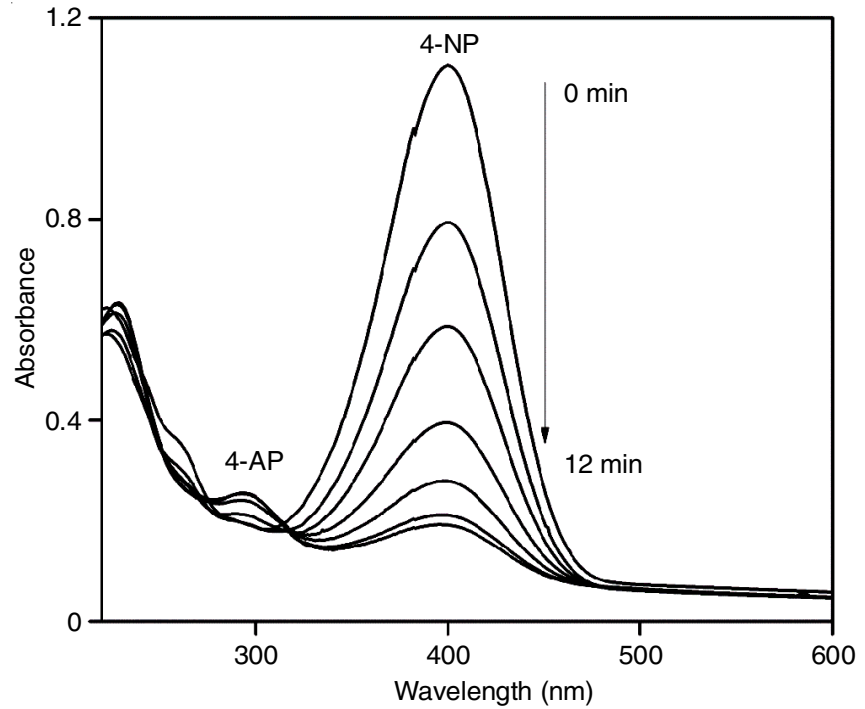

Fig. 8. UV-visible spectra of 4-nitrophenol (4-NP) using PS-PVBCPPI(G2)-AuNPs-H catalyst

AuNPs-L. This is because, as explained in characterization part, the former two catalysts must stabilized with more load of AuNPs due to availability of larger $-\mathrm{NH}_{2}$ group on the surface of beads matrices, obviously larger availability of $-\mathrm{NH}_{2}$ group on the surface is due to grafting of more glycidyl methacylate by ATRP method and thus enabled for more grafting of stabilizing agent viz., PPI(G2). Further, the stability of the superior catalyst PS-PVBC-PPI(G2)-AuNPs-H was examined by reusing the catalyst through filtration. The obtained $\mathrm{k}_{\mathrm{obs}}$ indicates that the activity is maintained even upto forth cycle and thus it is proved the catalyst is more stable and hence it can be more suitable for industrial reaction (Fig. 9d).

Kinetics of 4-nitrophenol using PS-PVPC-PPI(G2)AuNPs-H: In heterogeneous catalysis, the rate of the reaction generally increases linearly with the amount of the catalyst. With a view to examine the effect of PS-PVBC-PPI(G2)-AuNPs$\mathrm{H}$, the said reduction of 4-nitrophenol was performed by varying the catalyst amount from 2 to $10 \mathrm{mg}$ keeping the other parameters constant [32]. Plot derived from observed rate constant against the amount of catalyst is shown in Fig. 10. From this plot, it is understood that on increasing the amount of PS-PVBCPPI(G2)-AuNPs-H catalyst, the rate constant also increased in parallel (Fig. 9a). This is quite obvious that at higher catalyst amount, the active nanoparticle is contributed to enhanced rate constant. Meijboom and Antonels et al. [33] observed the same trend of results for the kinetic study of reduction of 4-nitrophenol using gold nano composites.

The effect of $\left[\mathrm{NaBH}_{4}\right]$ was also studied by fixing the concentration range from $0.6 \mathrm{M}$ to $1.4 \mathrm{M}$ keeping other parameters as constant. The pseudo-first order rate constants were evaluated and plotted against the $\left[\mathrm{NaBH}_{4}\right]$ (Fig. 11). The observed rate constants increased with the increasing the $\left[\mathrm{NaBH}_{4}\right]$ (Fig. 9b). Analogous trend in the rate constant with increasing $\left[\mathrm{NaBH}_{4}\right]$ was already reported [34]. On increasing the $\left[\mathrm{NaBH}_{4}\right]$, the adsorption of $\mathrm{NaBH}_{4}$ onto the nanoparticle surfaces also parallely increases, as a result, the release of electrons from $\mathrm{BH}_{4}{ }^{-}$

TABLE-2

KINETIC TABLE FOR 4-NITROPHENOL

\begin{tabular}{lccccc}
\hline \multirow{2}{*}{ Types of catalyst loading } & \multirow{2}{*}{$\mathrm{k}_{\mathrm{obs}} \times 10^{-3}\left(\mathrm{~s}^{-1}\right)$} & \multicolumn{3}{c}{$\mathrm{k}_{\mathrm{obs}} \times 10^{-3} \mathrm{~s}^{-1}$ for recycle catalyst } \\
\cline { 3 - 6 } & & Cycle-I & Cycle-II & Cycle-III & Cycle-IV \\
\hline PS-PVBC-PPI(G2)-AuNPs-L & 17.47 & - & - & - & 24.48 \\
PS-PVBC-PPI(G2)-AuNPs-H & 25.78 & 25.78 & - & - & - \\
PS-P4VP-PPI(G2)-AuNPs-L & 04.67 & - & - & - & - \\
PS-P4VP-PPI(G2)-AuNPs-H & 06.34 & - & - & - \\
\hline
\end{tabular}



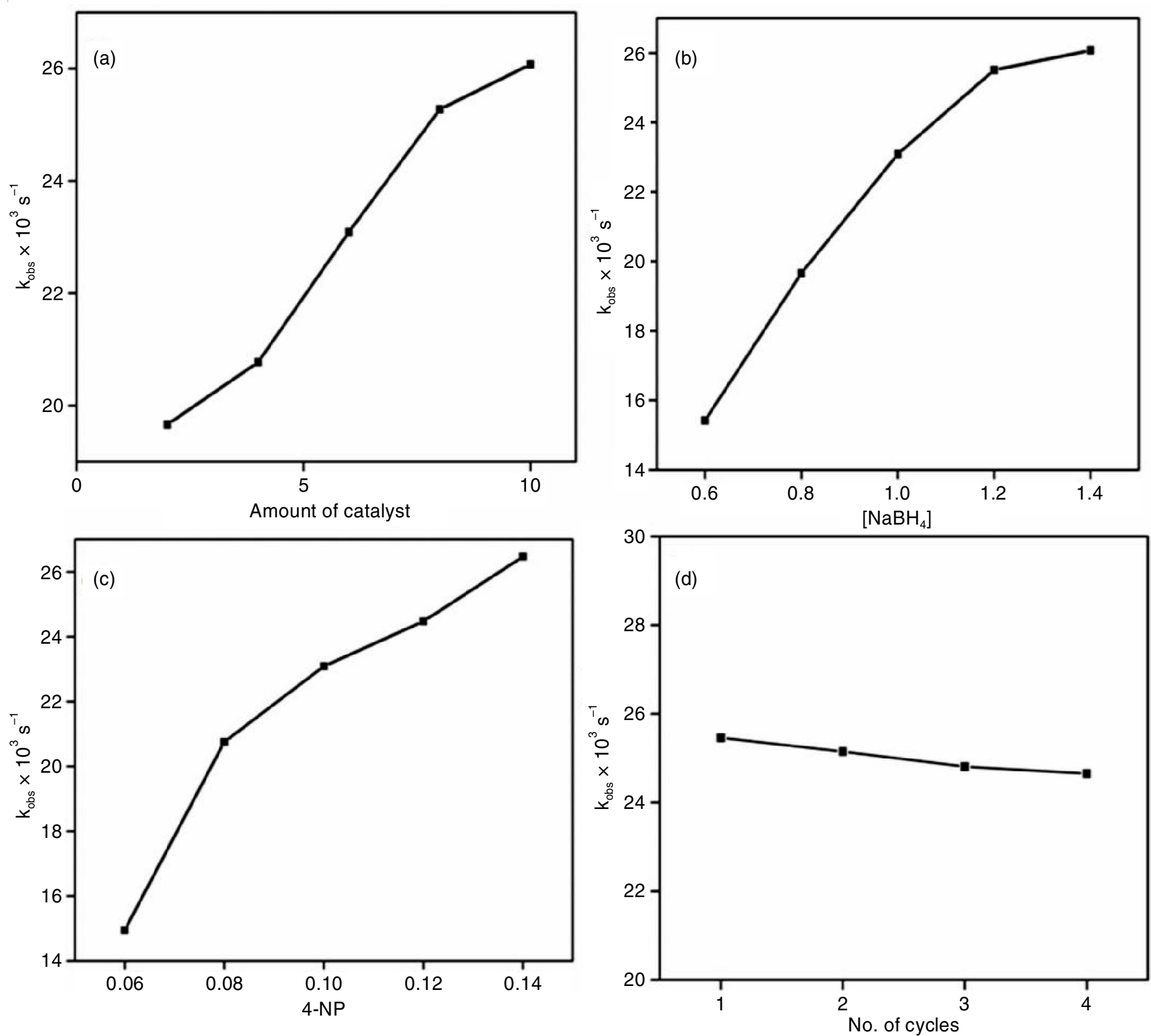

Fig. 9. Variation of (a) [catalyst], (b) $\left[\mathrm{NaBH}_{4}\right]$, (c) [4-NP], (d) recycling efficiency of PS-PVBC-PPI(G2)-AuNPs-H catalyst

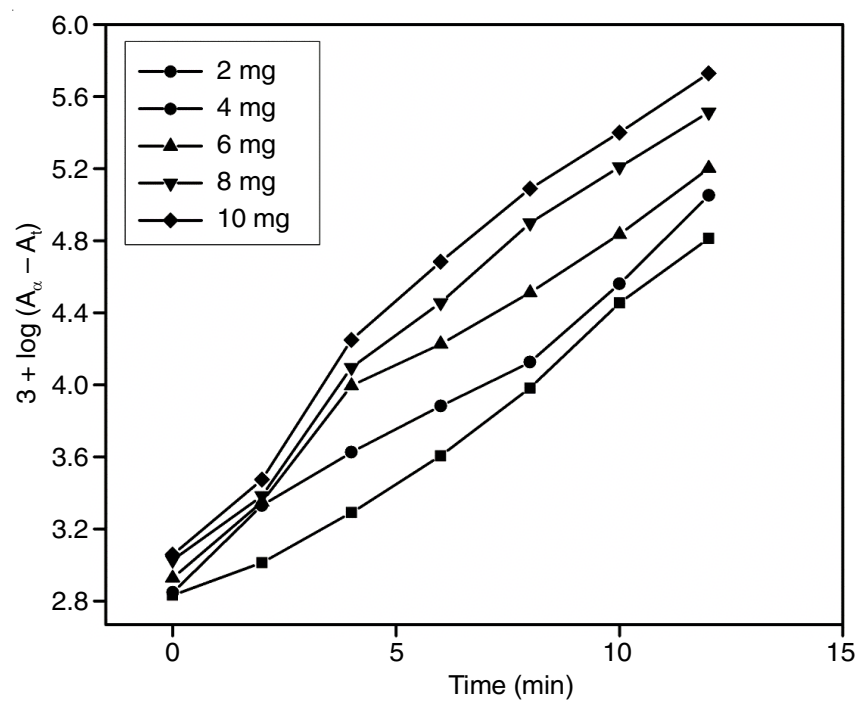

Fig. 10. Effect of catalyst

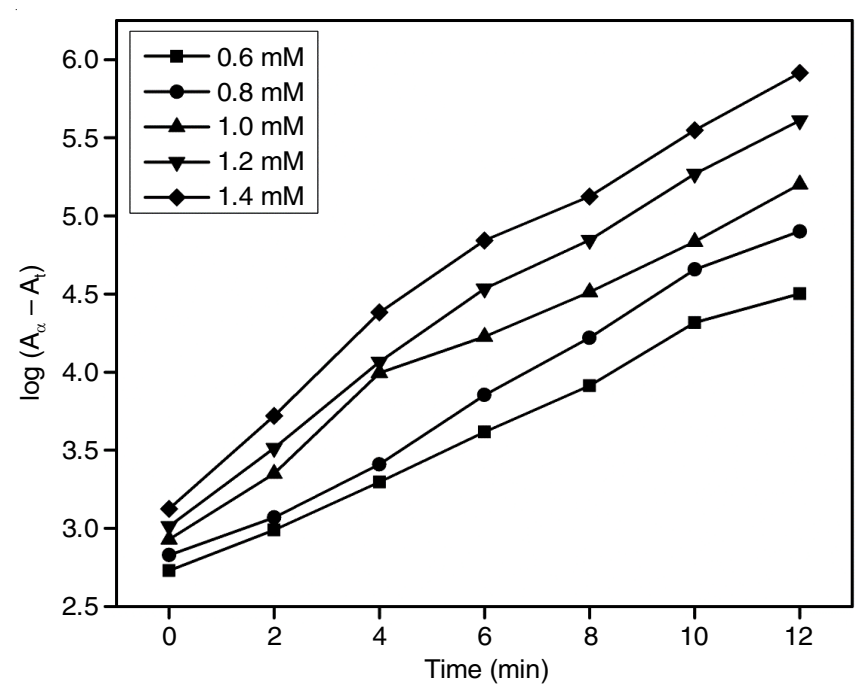

Fig. 11. Effect of $\left[\mathrm{NaBH}_{4}\right]$ 
(donor) to the 4-nitrophenol (receptor) increases [2]. Further, it is worth to state that under the fixed concentration of $\mathrm{NaBH}_{4}$ the reduced product viz., 4-amino phenol is not oxidized further because the liberated hydrogen from borohydride purged out the prevented the oxidation. In addition, evolution of small bubbles of hydrogen on the catalyst surface helped in mixing of the solution and thus offering the favorable conditions to increase the reaction rates.

In order to study the influence of [substrate] on the rate of reduction of 4-nitrophenol in the presence of PS-PVBCPPI(G2)-AuNPs-H catalyst and $\mathrm{NaBH}_{4}$ keeping the parameters as constant. The [4-nitrophenol] varied from $0.6 \times 10^{-2} \mathrm{M}$ to $1.4 \times 10^{-2} \mathrm{M}$. The pseudo-first order rate constants were calculated and plotted against the [substrate] from the plot (Fig. 12), it is revealed that the rate constant increased with the increase in the [4-nitrophenol] (Fig. 9c). Normally, the metallic nanoparticles strongly catalyze the reduction reactions by transferring electrons from $\mathrm{BH}_{4}{ }^{-}$ions [35] to the substrate after the adsorption of both substrate and $\mathrm{NaBH}_{4}$ ions onto the catalyst surface. In this study also, when the [substrate] increased, the probability of adsorption of more number of 4-nitrophenolate ions onto the catalyst surface was also parallelly increased and thus enhanced the rate constants. Added to this fact, $\mathrm{NaBH}_{4}$ present in aqueous medium is a strong nucleophile, because of its diffusive nature and high electron injection capability, transfers electron to the substrate via metal particles which in turn helps to overcome kinetic barrier for the reduction reaction to form 4-aminophenol. Similar, results on the rate of 4-nitrophenol reduction with addition of substrate were reported by Pal et al. [36].

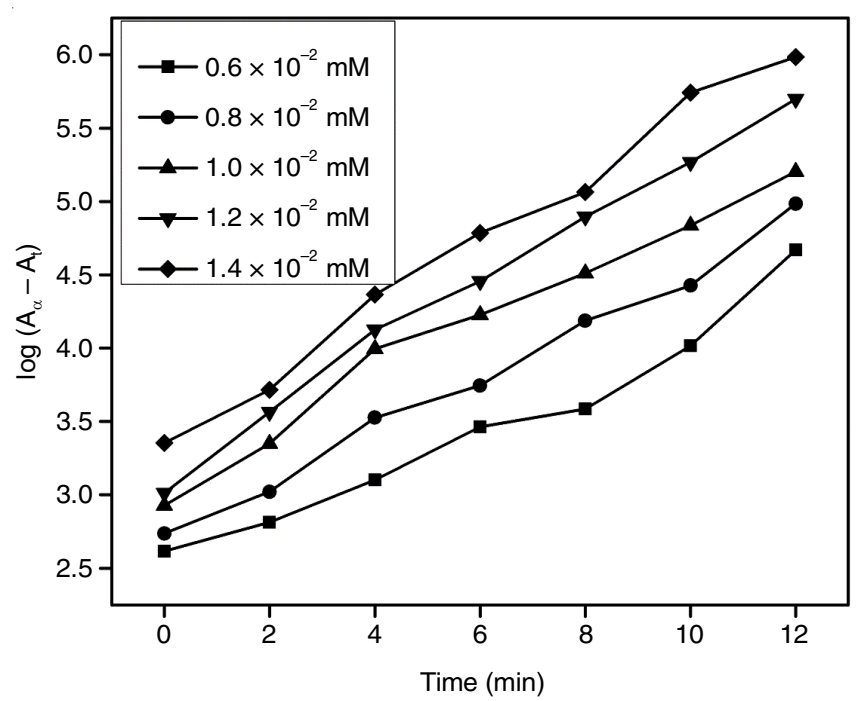

Fig. 12. Effect of [substrate]

\section{Conclusion}

Four types of polymer-supported beads grafted with dendrimer stabilized AuNPs catalysts viz., PS-PVBC-PPI(G2)AuNPs-L \& PS-PVBC-PPI(G2)-AuNPs-H and PSP4VP-PPI(G2)-AuNPs-L \& PS-P4VP-PPI(G2)-AuNPs-H were prepared by adopting simplified procedure. It is proved that the PSPVBC-PPI(G2) matrix [derived from PPI(G2) grafted on PSPVBC via SI-ATRP technique] was effective for efficient stabi- lization/immobilization of AuNPs than the PS-P4VP-PPI(G2) matrix (derived from grafting of PPI(G2) through quaternization technique). The formation of AuNPs onto the surface of both matrices was established by the appearance of SPR peak at $537 \mathrm{~nm}$ in UV and block dots observed in HRTEM images. In addition, higher absorbance in SPR peak and intense block dots in HRTEM were noticed in PS-PVBC-PPI(G2)AuNPs-L \& PS-PVBC-PPI(G2)-AuNPs-H than that of PSP4VP-PPI(G2)-AuNPs-L \& PS-P4VP-PPI(G2)-AuNPs-H. These heterogeneous nanoparticle catalysts were found to be efficient and reusable catalyst for the reduction of 4-nitrophenol under $\mathrm{NaBH}_{4}$ as a reducing agent. The catalyst remains stable and the catalytic activity was maintained even up to 4 cycle remains constant. The stability and catalytic activity was higher for the order of PS-PVBC-PPI(G2)-AuNPs-H > PS-PVBCPPI(G2)-AuNPs-L > PS-P4VP-PPI(G2)-AuNPs-H > PS-P4VPPPI(G2)-AuNPs-L, respectively. Since, SI-ATRP modified PPI(G2) dendrimer grafted polymer beads has availability of nanoparticle stabilized $-\mathrm{NH}_{2}$ much more than other types of polymer beads. The most important virtue of this catalyst is that since the catalyst is bead shaped, it can be very well packed in a column reactor and thus the reduction reaction can be conducted in continue mode operation in industries. Above all, the pollution that has been experienced through homogeneous nanoparticles catalysts or colloidal form of catalyst has been completely alleviated by substituting these heterogeneous nanoparticles catalysts for reduction reaction.

\section{ACKNOWLEDGEMENTS}

The authors gratefully acknowledged the National Natural Science Foundation (21607063, 51402130, 21676129), China Postdoctoral Science Foundation (2016M590421) and the Science \& Technology Foundation of Zhenjiang \& Yangzhou (GY2014028 and YZ2015019).

\section{CONFLICT OF INTEREST}

The authors declare that there is no conflict of interests regarding the publication of this article.

\section{REFERENCES}

1. M.A. Mahmoud and M.A. El-Sayed, J. Phys. Chem. A, 111, 17180 (2007); https://doi.org/10.1021/jp709735n.

2. S. Praharaj, S. Nath, S.K. Ghosh, S. Kundu and P. Pal, Langmuir, 20, 9889 (2004); https://doi.org/10.1021/la0486281.

3. A. Köckritz, M. Sebek, A. Dittmar, J. Radnik, A. Brückner, U. Bentrup, M.-M. Pohl, H. Hugl and W. Mägerlein, J. Mol. Catal. Chem., 246, 85 (2006); https://doi.org/10.1016/j.molcata.2005.10.020.

4. A.G. Boudjahem, S. Monteverdi, M. Mercy and M.M. Bettahar, J. Catal., 221, 325 (2004); https://doi.org/10.1016/j.jcat.2003.08.002.

5. D. Astruc, F. Lu and J.R. Aranzaes, Angew. Chem. Int. Ed., 44, 7852 (2005); https://doi.org/10.1002/anie.200500766.

6. B.M. Choudary, S. Madhi, N.S. Chowdari, M.L. Kantam and B. Sreedhar, J. Am. Chem. Soc., 124, 14127 (2002); https://doi.org/10.1021/ja026975w.

7. K. Mallick and M.S. Scurrell, J. Appl. Catal. A, 253, 527 (2003); https://doi.org/10.1016/S0926-860X(03)00552-0.

8. Z. Liu, J.Y. Lee, W. Chen, M. Han and L.M. Gan, Langmuir, 20, 181 (2004);

https://doi.org/10.1021/la035204i. 
9. K. Suzuki, T. Yumura, M. Mizuguchi, Y. Tanaka, C.W. Chen and M.J. Akashi, J. Appl. Polym. Sci., 77, 2678 (2000); https://doi.org/10.1002/1097-4628(20000919)77:12<2678::AIDAPP150>3.0.CO;2-9.

10. C.W. Chen, M.Q. Chen, T. Serizawa and M. Akashi, Chem. Commun., 831 (1998); https://doi.org/10.1039/a800203g.

11. C.W. Chen, T. Serizawa and M. Akashi, Chem. Mater., 11, 1381 (1999); https://doi.org/10.1021/cm9900047.

12. M.T. Greci, S. Pathak, K. Mercado, G.K.S. Prakash, M.E. Thompson and G.A. Olah, J. Nanosci. Nanotechnol., 1, 3 (2001); https://doi.org/10.1166/jnn.2001.001.

13. E. Murugan and J.N. Jebaranjitham, J. Mol. Catal. Chem., 365, 128 (2012); https://doi.org/10.1016/j.molcata.2012.08.021.

14. E. Murugan and R. Rangasamy, J. Polym. Sci. A Polym. Chem., 48, 2525 (2010); https://doi.org/10.1002/pola.24028.

15. R.G. Freeman, K.C. Grabar, K.J. Allison, R.M. Bright, J.A. Davis, A.P. Guthrie, M.B. Hommer, M.A. Jackson, P.C. Smith, D.G. Walter and M.J. Natan, Science, 267, 1629 (1995); https://doi.org/10.1126/science.267.5204.1629.

16. G. Chumanov, K. Sokolov, B.W. Gregory and T.M. Cotton, J. Phys. Chem., 99, 9466 (1995); https://doi.org/10.1021/j100023a025.

17. K.C. Grabar, R.G. Freeman, M.B. Hommer and M.J. Natan, Anal. Chem., 67, 735 (1995); https://doi.org/10.1021/ac00100a008.

18. W. Fritzsche, K. Sokolov, G.D. Chumanov, T.M. Cotton and E. Henderson, J. Vac. Sci. Technol., 14, 1766 (1996); https://doi.org/10.1116/1.580334.

19. M.M. Feldstein, V.G. Kulichikhin, S.V. Kotomin, T.A. Borodulina, M.B. Novikov, A. Roos and C. Creton, J. Appl. Polym. Sci., 100, 522 (2006); https://doi.org/10.1002/app.23290.

20. P. Liu, Y. Liu and Z. Su, Ind. Eng. Chem., 45, 2255 (2006); https://doi.org/10.1021/ie051247d.

21. R.B. Grubbs, C.J. Hawker, J. Dao and J.M. Frechet, Angew. Chem. Int. Ed. Engl., 36, 270 (1997);

https://doi.org/10.1002/anie.199702701.
22. P. Liu, Eur. Polym. J., 41, 2693 (2005); https://doi.org/10.1016/j.eurpolymj.2005.05.017.

23. T. Balakrishnan and E. Murugan, J. Polym. Sci. A Polym. Chem., 41, 347 (2003); https://doi.org/10.1002/pola.10562.

24. A. Murugan and P. Gopinath, J. Appl. Catal. A, 319, 72 (2007); https://doi.org/10.1016/j.apcata.2006.11.012.

25. Y. Du, H. Chen, R. Chen and N. Xu, J. Appl. Catal. A, 277, 259 (2004); https://doi.org/10.1016/j.apcata.2004.09.018.

26. M.J. Vaidya, S.M. Kulkarni and R.V. Chaudhari, Org. Process Res. Dev., 7, 202 (2003); https://doi.org/10.1021/op025589w.

27. P.C. Kearney and D.D. Kaufman, Herbicides: Chemistry, Degradation and Mode of Action, Marcel Dekker, Inc.: New York (1976).

28. N.G. McCormick, F.E. Feeherry and H.S. Levinson, Appl. Environ. Microbiol., 31, 949 (1976).

29. H. Terada, Biochim. Biophys. Acta, 639, 225 (1981); https://doi.org/10.1016/0304-4173(81)90011-2.

30. T. Balakrishnan and W.T. Ford, J. Appl. Polym. Sci., 27, 133 (1982); https://doi.org/10.1002/app.1982.070270115.

31. S. Panigrahi, S. Basu, S. Praharaj, S. Pande, S. Jana, A. Pal, S.K. Ghosh and T. Pal, J. Phys.Chem. C, 111, 4596 (2007); https://doi.org/10.1021/jp067554u.

32. Y.-C. Chang and D.-H. Chen, J. Hazard. Mater, 165, 664 (2009); https://doi.org/10.1016/j.jhazmat.2008.10.034.

33. N.C. Antonels and R. Meijboom, Langmuir, 29, 13433 (2013); https://doi.org/10.1021/la402885k.

34. A. Henglein, Chem. Rev., 89, 1861 (1989); https://doi.org/10.1021/cr00098a010.

35. K.S. Shin, J.Y. Choi, C.S. Park, H.J. Jang and K. Kim, Catal. Lett., 133, 1 (2009); https://doi.org/10.1007/s10562-009-0124-7.

36. S.K. Ghosh, M. Mandal, S. Kundu, S. Nath and T. Pal, Appl. Catal A, Gen., 268, 61 (2004);

https://doi.org/10.1016/j.apcata.2004.03.017. 